\title{
Study on Capacity Expansion Problem of Minimum Cost Flow \\ Geng Liu
}

\author{
School of Economics and Management, China University of Geosciences, Wuhan Hubei China, \\ 430074. \\ liujiangpin@263.net
}

\begin{abstract}
Keywords: Directed networks; Minimum cost flow problem; Polynomial algorithm; Capacity expansion
\end{abstract}

\begin{abstract}
The minimum cost flow problem is the central object of study in the network flows. This paper studies capacity expansion problem of minimum cost flow. How to expand the network capacity effectively so that the network flow can reach a certain level with minimum total cost? In view of three types of expanding models: the arc-expanding model, the node-expanding model and the combination of arc-node expanding model; this article discusses the characteristics of the problems separately and a unified capacity expansion model is also proposed. Finally, an example has been provided in detail.
\end{abstract}

\section{Introduction and Motivation}

Capacity expansion can be seen almost everywhere in the world. For example, in order to increase the flow ability of a road network to a certain level, we wish to expand the road capacity. Similar expansion occurs to transportation system or telecommunication network.

So far, several works have been done. Omar J. Guerra, Diego A. Tejada and Gintaras V. Reklaitis [1] presented a comprehensive optimization framework for the design and planning of interconnected power systems, including the integration of generation and transmission capacity expansion planning. Sinan Gürel [2] considered a multicommodity network flow problem with flow routing and discrete capacity expansion decisions. Miguel Gueifão Santos and António Pais Antunes [3] introduced an optimization model aimed to assist aviation authorities in their strategic decisions regarding the long-term expansion of a network of airports. RL Burdett [4] considered the expansion of railways in order to increase their capacity. Ahuja and Orlin [5] considered how to improve the capacity of network to send flow as much as possible. G.N.Frederickson [6] considered the problems of increasing the weight of minimum spanning tree. C. Yang and G. Liu [7] studied a model for multiperiod capacity expansion problems on networks. L.P. Wang, S. Z. Wang and G.H. $\mathrm{Xu}$ [8] introduced a model for the network capacity expansion with the time and cost constraints. C.Yang. and J.Zhang [9] studied the bottleneck capacity expansion problems on networks. J. Yang and C. Yang [10] considered a model of flow capturing location-allocation problem with chance constraint. H. C. Yan, X.H. Huang and M. Wang [11] dealed with the problem of delay-dependent stability for networked control systems with multiple time- delays.

In this article, we assume a directed network $\mathrm{G}(\mathrm{V}, \mathrm{A}, \mathrm{C}, \mathrm{B}, \mathrm{F})$ consisting of a set of nodes $\mathrm{V}=\left\{v_{1}, v_{2}, \ldots, v_{n}\right\}$, a set of arcs $A \subseteq\left\{\left(v_{i}, v_{j}\right) \mid i=1,2, \cdots, n, j=1,2, \cdots, n\right\}$, a capacity vector $\mathrm{C}$, a cost vector $\mathrm{B}$ and a network flow vector $\mathrm{F}$. The component $c_{i j}$ of $\mathrm{C}$ denotes the capacity of arc $\left(v_{i}, v_{j}\right)$. The component $b_{i j}$ of $\mathrm{B}$ denotes the unit cost of arc $\left(v_{i}, v_{j}\right)$. The component $f_{i j}$ of $\mathrm{F}$ denotes the flow of $\operatorname{arc}\left(v_{i}, v_{j}\right)$. The source node is $v_{s}$ and sink node is $v_{t}$.

There Are Three Types of Expanding Models. The Arc-Expanding Model. In this model, the unit cost to increase the capacity of arc $\left(v_{i}, v_{j}\right)$ is $\omega_{i j}$. The network capacity is increased by the expansion of arcs. This model is widely used. The path widening belongs to this kind of expanding model.

The Node-Expanding Model. On this model, it is assumed that the capacities of all arcs 
$\left(v_{i}, v_{j}\right)$ which start at the same node $v_{i}$ should be increased by the same amount and that the unit cost to make such expansion is $\omega_{i}$. For example, the installation of new concentrators at the nodes of a local access telecommunications network can be viewed as this kind of expanding model.

The Combination of Node-Expanding and Arc-Expanding Model. In the process of network adjustment, the two models may carry on simultaneity. For example, in the telecommunications industry, capacity expansion can be realized through the installation of new concentrators at the nodes and replacing existing links with new type of links.

\section{Problem Formulation}

Given a directed network G ( V, A, C, B, F) defined as above. Let $\alpha_{i}$ be the amount for expansion capacity on node $v_{i}, \alpha_{i j}$ be the amount for expansion capacity on arc $\left(v_{i}, v_{j}\right), \mathrm{R}$ be a given flow value that the network should be enhanced to, $\mathrm{D}$ be a given budget.

We also assume that the capacity and flow on every arc be integers and the optimal solutions be integers, too.

The capacity expansion problem can be stated as follows: for a given value $\mathrm{R}$, how can the flow of minimum cost flow be increased to $\mathrm{R}$ with a minimum total cost?

To Solve This Problem, We Will Discuss It on the Three Expanding Models. On the arc-expanding model, the problem can be stated as (LP1):

$$
\begin{aligned}
& \min \left(\sum_{\left(v_{i}, v_{j}\right) \in V} b_{i j} f_{i j}+\sum_{\left(v_{i}, v_{j}\right) \in V} \omega_{i j} \alpha_{i j}\right) \\
& \text { s.t. } \sum f_{i j}-\sum f_{j i}= \begin{cases}R, & i=s \\
-R, & i=t \\
0, & \text { otherwise }\end{cases} \\
& 0 \leq f_{i j} \leq c_{i j}+\alpha_{i j} \\
& \alpha_{i j} \geq 0, f_{i j} \geq 0
\end{aligned}
$$

This is a linear programming. The objective function states that we want to minimize the sum of the expanding and transportation cost. We refer to the constraints (2) as mass balance flow bound constraints, constraints (3) as flow bound constraints, and constraints (4) as nonnegative constraints.

On the arc-expanding model, the capacity expansion problem can be transformed into a minimum cost flow problem with flow value $\mathrm{R}$ in an auxiliary network $G^{\prime}\left(V, A^{*}, C^{\prime}, B^{\prime}\right)$ as follows: for each arc $\quad\left(v_{i}, v_{j}\right) \in A$, we have a second arc $\left(v_{i}, v_{j}\right)^{\prime}$. Let $A^{\prime}$ be the set of all second arcs and $A^{*}=A \cup A^{\prime}$. The capacity vector $C^{\prime}$ is defined by

$$
c^{\prime}{ }_{i j}=\left\{\begin{array}{l}
+\infty, \forall\left(v_{i}, v_{j}\right)^{\prime} \in A^{\prime} ; \\
c_{i j}, \forall\left(v_{i}, v_{j}\right) \in A
\end{array}\right.
$$

And the cost vector is defined by

$$
b_{i j}^{\prime}=\left\{\begin{array}{l}
b_{i j}, \forall\left(v_{i}, v_{j}\right) \in A ; \\
b_{i j}+\omega_{i j}, \forall\left(v_{i}, v_{j}\right)^{\prime} \in A^{\prime}
\end{array}\right.
$$

Now we find out a minimum cost flow $f^{*}$ from $v_{s}$ to $v_{t}$ with a total flow value $\mathrm{R}$ in network $G^{\prime}\left(V, A^{*}, C^{\prime}, B^{\prime}\right)$, which can be written as follows (LP1-1):

$$
\min \sum_{\left(v_{i}, v_{j}\right) \in A^{*}} b_{i j}^{\prime} f_{i j}
$$


s.t. $0 \leq f_{i j} \leq c^{\prime}{ }_{i j}, \forall\left(v_{i}, v_{j}\right) \in A^{*}$,

$\sum_{j} f_{i j}-\sum_{j} f_{j i}=\left\{\begin{array}{l}R, \text { if } i=s ; \\ -R, \text { if } i=t ; \\ 0, \text { otherwise }\end{array}\right.$

The problem can be solved by strong polynomial algorithms and the optimal flow $f_{i j}^{*}$ on the arc $\left(v_{i}, v_{j}\right)^{\prime}$ corresponds to the capacity value which we need to increase on arc $\left(v_{i}, v_{j}\right)$. The total cost for expansion and transportation is the objective value of (LP1-1), that is, the minimum cost of flow $\mathrm{f}^{*}$ on $G^{\prime}\left(V, A^{*}, C^{\prime}, W^{\prime}\right)$. Therefore, the problem (LP1) can be solved by strong polynomial algorithm.

On the node-expanding model, the problem can be expressed as (LP2):

$$
\begin{aligned}
& \min \left(\sum_{\left(v_{i}, v_{j}\right) \in V} b_{i j} f_{i j}+\sum_{i=1}^{n} \omega_{i} \alpha_{i}\right) \\
& \sum f_{i j}-\sum f_{j i}=\left\{\begin{array}{l}
R, i=s ; \\
-R, i=t ; \\
0, \text { othwise }
\end{array}\right. \\
& 0 \leq f_{i j} \leq \alpha_{i}+c_{i j} \\
& \alpha_{i} \geq 0, f_{i j} \geq 0
\end{aligned}
$$

This is a linear programming. The objective function states that we want to minimize the sum of node-expanding and transportation cost. The constraints (11), (12), (13) are the same as (LP1).

On the combination of node-expansion and arc expansion model, the problem can be stated as (LP3):

$$
\begin{aligned}
& \min \left(\sum_{\left(v_{i}, v_{j}\right) \in V} b_{i j} f_{i j}+\sum_{\left(v_{i}, v_{j}\right) \in A} \omega_{i j} \alpha_{i j}+\sum_{v_{i} \in V} \omega_{i} \alpha_{i}\right) \\
& \sum_{j} f_{i j}-\sum_{j} f_{j i}=\left\{\begin{array}{l}
R, \text { if } i=s ; \\
-R, \text { if } i=t ; \\
0, \text { otherwise }
\end{array}\right. \\
& 0 \leq f_{i j} \leq \alpha_{i}+\alpha_{i j}+\underline{c}_{i j} \\
& \alpha_{i j} \geq 0, f_{i j} \geq 0
\end{aligned}
$$

This is a linear programming. The objective function states that we want to minimize the sum of expanding and transportation cost. The constraints (15), (16), (17) are the same as (LP1).

We can see: (LP1) and (LP2) are all special cases of (LP3). We only consider one type of expanding model on (LP1) and (LP2). That is, the general expanding type is the combination of node-expansion and arc expansion model. So, (LP3) is the general model.

\section{Case Study}

Given a directed network G(V, A, C, B, F) described in Fig.1. The first number in brackets besides every arc is the capacity $c_{i j}$ of that arc and the second is the unit cost $b_{i j}$ of that arc. It is easy to know the present maximum flow is 5 . The unit cost $\omega_{i j}$ for arc-expanding and $\omega_{i}$ for nodeexpanding are shown in Table 1 and 2 respectively. 
The problem is: for a given value $\mathrm{R}=8$, how can the flow of minimum cost flow be enhanced to 8 with minimum total cost?

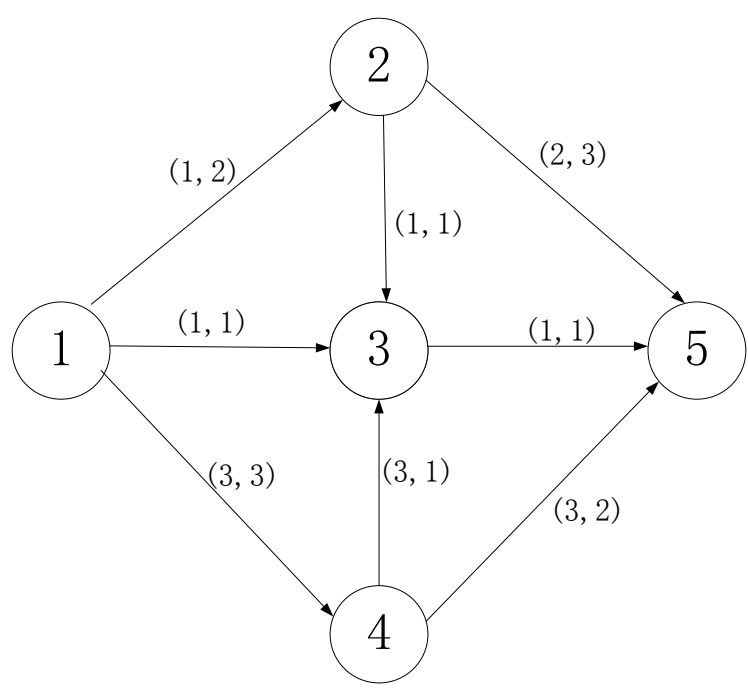

Figure 1. Network for Expansion

Table 1 The unit cost to increase the capacity of arcs

\begin{tabular}{ccccccccc}
\hline $\operatorname{Arcs}$ & $(1,2)$ & $(1,3)$ & $(1,4)$ & $(2,3)$ & $(2,5)$ & $(3,5)$ & $(4,3)$ & $(4,5)$ \\
\hline$\omega_{i j}$ & 3 & 2 & 4 & 2 & 2 & 3 & 2 & 1 \\
\hline
\end{tabular}

Table 2 The unit cost to increase the capacity of nodes

\begin{tabular}{clllll}
\hline Nodes & 1 & 2 & 3 & 4 & 5 \\
\hline$\omega_{i}$ & 1 & 3 & 4 & 1 & 5 \\
\hline
\end{tabular}

As stated above, we establish mathematical formulations separately. We solve the problem with Excel. The results are shown below.

On the arc-expanding model, the total cost is 43 . The expansion arcs are $(1,3)$ and $(3,5)$, the expansion capacity are both 3 .

On the node-expanding model, the minimum cost is 40 . The expansion nodes are 1,3 and 4 , the expansion capacity are all 1.

On the combination of node-expanding and arc-expanding model, the minimum cost is 39 . The expanding arc is $(3,5)$ and expansion capacity is 1 . The expansion node is 1 and 4 . The expansion capacity are both1.

The results show: on the combination of node-expanding and arc-expanding model, the expansion cost is the lowest among the three models.

\section{Conclusion}

Either node-expanding model or arc-expanding one has its own superiority. If they could be combined properly, a better result can be reached. We also point out: the combination of node-expansion and arc expansion model is the general expanding type. Under the foundation of separate discussion, we establish a general linear programming model. This makes the model more practical. Possible extension of this paper would be to allow arbitrary demand. A more general model is currently under investigation. 


\section{References}

[1] Omar J. Guerra, Diego A. Tejada and Gintaras V. Reklaitis. An optimization framework for the integrated planning of generation and transmission expansion in interconnected power systems. Applied Energy 170(2016): 1-21

[2] Sinan Gürel. A conic quadratic formulation for a class of convex congestion functions

[3] In network flow problems. European Journal of Operational Research 211 (2011):252-22

[4] Miguel Gueifão Santos, António Pais Antunes. Long-term evolution of airport networks: Optimization model and its application to the United States. Transportation Research Part E 73(2015): 17-46

[5] RL Burdett. Optimisation models for expanding a railway's theoretical capacity. European Journal of Operational Research 251 (2016): 783-797

[6] R. K. Ahuja, T. L. Magnanti and J. B. Orlin, Network Flows, Prentice-Hall, Englewood Cliffs, New Jersey, 1993.

[7] Frederickson G N. Increasing the weight of minimum spanning tree. In: Proceedings of the 7th Annual ACM-SLAM Symposium on Discrete Algorithm (SODA'96), Jan, 1996. 539-546

[8] C. Yang and G. Liu. A model for multiperiod capacity expansion problems on networks. Journal of Systems Science and Information, 2003. Vol. 1, No. 4, 525-529.

[9] L.P. Wang, S.Z. Wang and G.H. Xu. The network capacity expansion problem with the time and cost constraints. Computer Engineering and Applications, Vol. 11:176-178, 2003.

[10]C. Yang and J.Z. Zhang. On the bottleneck capacity expansion problems on networks. Acta Mathematica Scientia 2006, 26B(2):202-208

[11][10] J. Yang, C. Yang and C.Y. Liu. Model and Algorithm of Flow Capturing Location-Allocation Problem with Chance Constraint. Advances in Systems Science and Applications, 2006, Vol.6, No.1:75-81

[12]H.C. Yan, X.H. Huang and M. Wang. Delay-dependent Stability Criteria for a Class of Networked Control Systems with Multiple Time-delays. Advances in Systems Science and Applications, 2006, Vol.6, No.2:184-192 